\title{
L'IMPACT DES RÉFORMES DE LA FORMATION EN TRAVAIL SOCIAL EN SUISSE : REGARDS CROISÉS DES PROFESSIONNELS DU HANDICAP
}

\author{
Valérie Perriard et Alida Gulfi
}

Céreq | « Formation emploi »

2019/4 n 148 | pages 69 à 85

ISSN 0759-6340

Article disponible en ligne à l'adresse :

https://www.cairn.info/revue-formation-emploi-2019-4-page-69.htm

Distribution électronique Cairn.info pour Céreq.

(C) Céreq. Tous droits réservés pour tous pays.

La reproduction ou représentation de cet article, notamment par photocopie, n'est autorisée que dans les limites des conditions générales d'utilisation du site ou, le cas échéant, des conditions générales de la licence souscrite par votre établissement. Toute autre reproduction ou représentation, en tout ou partie, sous quelque forme et de quelque manière que ce soit, est interdite sauf accord préalable et écrit de l'éditeur, en dehors des cas prévus par la législation en vigueur en France. Il est précisé que son stockage dans une base de données est également interdit. 
FORMATION Formation emploi

Revue française de sciences sociales

148 | Octobre-Décembre

Varia

\section{L'impact des réformes de la formation en travail social en Suisse : regards croisés des professionnels du handicap}

The impact of social work training reforms in Switzerland : perspective of professionals working in the field of disability Auswirkungen der Reform der Ausbildung in sozialer Arbeit in der Schweiz: Wahrnehmungen der Fachleute im Behindertenbereich El impacto de las reformas de la formación en trabajo social en Suiza : miradas cruzadas de los profesionales de la discapacidad

\section{Valérie Perriard et Alida Gulfi}

\section{OpenEdition}

Journals

Édition électronique

URL : http://journals.openedition.org/formationemploi/7830

ISSN : 2107-0946

\section{Éditeur}

La Documentation française

\section{Édition imprimée}

Date de publication : 31 décembre 2019

Pagination : 69-85

ISSN : 0759-6340

Distribution électronique Cairn

\section{CAIRN INEO}

CHERCHER, REPÉRER, AVANCER

Référence électronique

Valérie Perriard et Alida Gulfi, «L'impact des réformes de la formation en travail social en Suisse: regards croisés des professionnels du handicap ». Formation emploi [En ligne], 148 | OctobreDécembre, mis en ligne le 04 janvier 2021, consulté le 12 décembre 2019. URL : http:// journals.openedition.org/formationemploi/7830

Ce document a été généré automatiquement le 12 décembre 2019.

(C) Tous droits réservés 


\title{
L'impact des réformes de la formation en travail social en Suisse : regards croisés des professionnels du handicap
}

\author{
The impact of social work training reforms in Switzerland : perspective of \\ professionals working in the field of disability \\ Auswirkungen der Reform der Ausbildung in sozialer Arbeit in der Schweiz: \\ Wahrnehmungen der Fachleute im Behindertenbereich \\ El impacto de las reformas de la formación en trabajo social en Suiza : miradas \\ cruzadas de los profesionales de la discapacidad
}

Valérie Perriard et Alida Gulfi

En Suisse, comme dans d'autres pays européens, le système de formation en travail social a connu de profondes transformations au cours des quinze dernières années, suite à une restructuration de la formation professionnelle et des formations dispensées au sein des hautes écoles. Ces transformations ont inclus une multiplication de formations et niveaux de diplôme, ainsi que leur reconnaissance sur le plan national. Actuellement, dix types de titres différents sont délivrés en travail social, et ce, à tous les niveaux de formation: au degré secondaire II (attestation fédérale de formation professionnelle, certificat fédéral de capacité, maturité professionnelle et maturité spécialisée), ainsi qu'aux niveaux supérieurs B (diplôme fédéral, brevet fédéral et diplôme d'école supérieure ES) et A (bachelor, master et doctorat) (Keller, 2017).

Dans le champ plus spécifique de l'éducation sociale ${ }^{1}$, plusieurs formations, de degrés différents, ont pris le relais de celle, traditionnelle, d'éducateur spécialisé, contribuant à une diversification des profils de formation et des niveaux de qualification des professionnels exerçant dans les institutions socio-éducatives.

Cet article présente quelques résultats d'une recherche conduite en Suisse romande, sur la collaboration, dans les institutions résidentielles du handicap, entre des 
professionnels issus de trois niveaux de formation : des assistants socio-éducatifs (ASE), des éducateurs sociaux ES et des éducateurs sociaux $\mathrm{HES}^{2}$.

La formation d'ASE a vu le jour dès 2005-2006. De niveau secondaire (certificat fédéral de capacité), accessible directement au terme de l'école obligatoire (dès l'âge de 15 ans), il s'agit de la première formation de type apprentissage disponible dans le domaine du travail social en Suisse romande. Au niveau supérieur, la formation d'éducateur social ES, dispensée par les écoles supérieures, est une formation spécialisée. Quant à la formation bachelor en travail social, elle a été instaurée en hautes écoles spécialisées (HES) à partir de 2002-2003. De type généraliste, elle comporte plusieurs orientations, dont une en éducation sociale.

5 Ces trois niveaux de formation sont théoriquement supposés constituer des profils professionnels distincts, disposant chacun de leur aire spécifique de responsabilités, activités et compétences (CDIP, 1999). L'assistant socio-éducatif (ASE) est censé agir de manière encadrée, sous la responsabilité des professionnels de formation supérieure (éducateurs sociaux ES et HES), et assumer des activités d'accompagnement au quotidien des usagers. L'éducateur social ES est présenté comme un professionnel autonome, qui prend en charge de façon indépendante des situations complexes, mais structurées, et exerce des responsabilités dans des domaines précis. L'éducateur social HES est décrit comme un expert, qui gère des situations de travail complexes, peu structurées, et assure une fonction d'encadrement d'équipe et d'évaluation scientifique.

6 Si ces divers profils professionnels sont décrits et clairement différenciés d'un point de vue théorique, comment s'opère leur articulation dans la pratique ? La question suscite de nombreuses discussions au sein des milieux professionnels et de la formation, qui soulèvent des enjeux relatifs à la professionnalisation du domaine, aux frontières et zones de tuilage entre les professions, ainsi qu'aux rapports entre ces professionnels. Néanmoins, les études empiriques qui l'abordent sont rares; dès lors, il nous a paru pertinent de réaliser une recherche visant à mieux appréhender comment se développe la collaboration entre ASE, éducateurs sociaux ES et éducateurs sociaux HES dans les institutions résidentielles du handicap, en Suisse romande, ainsi que les enjeux qu'elle recèle. La présence de ces trois groupes professionnels implique-t-elle des réaménagements organisationnels, notamment en matière de recrutement, de composition des équipes et de division du travail entre ASE, éducateurs sociaux ES et HES ? Quelles sont les responsabilités et activités de ces professionnels? Comment se développe leur collaboration au sein des équipes éducatives et quels en sont les points forts et les limites?

7 Soulignons que cette recherche a été menée entre 2014 et 2016, alors que les restructurations de la formation en travail social étaient récentes et les titres encore peu stabilisés et connus. Les nouveaux diplômés étaient fraîchement arrivés sur le marché du travail, la cohabitation dans les structures du handicap entre ASE, éducateurs sociaux ES et éducateurs sociaux HES était nouvelle et les réflexions sur les terrains largement en cours quant à l'articulation de ces différents profils.

8 La question de la collaboration, dans les institutions du domaine du handicap, entre ASE, éducateurs sociaux ES et éducateurs sociaux HES a été appréhendée sous l'angle de la sociologie des professions, et plus particulièrement de la perspective dite des groupes professionnels (Bercot, Divay \& Gadéa, 2012 ; Demazière \& Gadéa, 2009 ; Dubar, Tripier \& Boussard, 2011). Ce courant, qui s'inscrit dans une approche interactionniste et privilégie l'idée de construction sociale, envisage lesdits groupes comme des 
processus interactifs et évolutifs "vulnérables, ouverts, instables " (Demazière \& Gadéa, op. cit., p. 20), «dont le devenir, jamais acquis, se joue en tension entre des forces internes et externes» (Bercot \& al., 2012, p.2). La recherche sociologique sur les groupes professionnels analyse ainsi principalement leur dynamique, c'est-à-dire les processus par lesquels les activités professionnelles émergent, se transforment, se différencient, voire disparaissent (Bercot \& al., op. cit. ; Demazière \& Gadéa, op. cit. ; Dubar \& al., op. cit. ). Elle explore en particulier les mécanismes de recomposition de la division du travail, les mouvements dans les attributions (des responsabilités, activités, compétences, rôles, etc.), ainsi que les déplacements dans les spécialisations professionnelles. Elle investigue également les relations de concurrence et de coopération entre les groupes professionnels, de même que les régulations formelles et informelles mises en œuvre par les acteurs pour contrôler, défendre, voire étendre leur territoire (Abbot, 1988).

Prenant appui sur cette recherche, l'article présente et discute les logiques institutionnelles à l'œuvre en matière de division du travail entre ASE, éducateurs sociaux ES et éducateurs sociaux HES. Il éclaire la manière dont les professionnels se positionnent face à ces logiques et se les approprient. Un regard est également porté sur la qualité perçue de la collaboration entre ces personnels et les défis à relever pour l'avenir de leur cohabitation.

\section{Encadré 1. Repères méthodologiques}

Sur le plan méthodologique, la recherche a été menée en deux étapes. Dans un premier temps, un sondage par questionnaire a été adressé à l'ensemble des structures résidentielles francophones $(\mathrm{N}=53)$ accompagnant des personnes de tout âge en situation de handicap mental, physique, psychique et/ou polyhandicap, dans deux cantons de Suisse romande, Fribourg $(\mathrm{N}=21)$ et Vaud $(\mathrm{N}=32)$. Le but de ce sondage était d'identifier les institutions comptant des équipes éducatives mixtes, c'est-à-dire composées à la fois d'assistants socioéducatifs (ASE), d'éducateurs sociaux diplômés d'une école supérieure (ES) et d'éducateurs sociaux titulaires d'un Bachelor d'une haute école spécialisée (HES). Sur les 39 institutions qui ont retourné le questionnaire rempli (taux de réponse de $74 \%), 19$ (49\%) ont mentionné compter des équipes mixtes.

Dix-huit de ces dix-neuf structures ont accepté de prendre part à la seconde phase de l'étude, qui a consisté en la réalisation d'entretiens individuels. 46 entretiens de type semi-structuré ont été menés avec les personnels concernés et les directions exerçant dans ces institutions : ASE (11 entretiens), éducateurs sociaux ES (9), éducateurs sociaux HES (13), directions d'institutions (13) $\left(^{*}\right)$. Les participants ont été recrutés sur la base du volontariat.

Conformément à la féminisation des professions du domaine social, plus de femmes $(n=28)$ que d'hommes $(n=20)$ ont participé à l'entretien. L'âge moyen des participants est de 37 ans (écart-type $=10.36$, étendue allant de 21 à 56 ans) et la plupart $(\mathrm{n}=41)$ sont de nationalité suisse.

Deux guides d'entretiens différents ont été élaborés, l'un à l'intention des professionnels, l'autre à celle des directions. Les thèmes abordés ont été identiques, mais les questions ont parfois été quelque peu adaptées. En lien avec les objectifs de l'étude, les thématiques suivantes ont été explorées : (a) le contexte professionnel et la composition des équipes, notamment la répartition entre les trois profils professionnels ; (b) les responsabilités, activités et compétences des ASE, des éducateurs sociaux ES et des éducateurs sociaux HES ; (c) l'attribution des 
responsabilités et activités et les aspects du cadre prescriptif (fonction, cahiers des charges, salaires, position dans l'organigramme institutionnel) ; (d) la collaboration entre ASE, éducateurs sociaux ES et éducateurs sociaux HES ; finalement (e) les représentations des acteurs de l'avenir des trois professions et de leur collaboration. Les entretiens ont duré, en moyenne, $1 \mathrm{~h} 15$. Ils ont été enregistrés, avec l'accord des participants.

Les données recueillies ont été soumises à une analyse de contenu thématique classique (Bardin, 2007 ; Miles \& Huberman, 2003) : retranscriptions intégrales, pré-analyse, construction des thématiques d'analyse, codage, analyse et interprétation des données. Un codage mixte hypothético-déductif et inductif a été effectué. Des synthèses par entretien ont d'abord été réalisées, puis des analyses transversales, comparant les réponses et relevant les similitudes et différences entre les institutions participantes et les quatre groupes d'acteurs interviewés. $\left(^{*}\right)$ : deux entretiens avec les directions ont été menés en binôme.

\section{Les logiques institutionnelles de division du travail : indifférenciation versus différenciation}

Deux logiques de division du travail se développent dans les structures du handicap consultées. L'une, que nous avons dénommée l'indifférenciation, concerne un peu plus de la moitié des établissements participants. Elle consiste à ne pas distinguer, sur le plan formel, entre les trois profils professionnels: ASE, éducateurs sociaux ES et éducateurs sociaux HES sont engagés sur la fonction d'éducateur; ils partagent un même cahier des charges, avec des responsabilités et activités prescrites qui sont identiques :

"C'est vrai qu'on a pris le pari d'avoir un cahier des charges qui est unique, la personne est engagée comme éducateur ou comme éducatrice, indépendamment de son niveau de formation, elle a le même cahier des charges. " "(Direction, homme, 38 ans)

11 L'autre logique, appelée la différenciation, implique une distinction entre les ASE, qui sont engagés comme tels ou sur la fonction d'éducateur auxiliaire, et les éducateurs sociaux ES et HES, qui sont employés comme éducateurs, avec un même cahier des charges pour les deux niveaux de formation.

"Oui, y'a le cahier des charges pour les éducateurs sociaux, pas spécifié HES ou ES, c'est juste "éducateurs sociaux». Y'a le cahier des charges des ASE. » (Éducateur social ES, femme, 32 ans)

Ces logiques s'observent dans les deux cantons explorés, dans des structures de taille variable, prenant en charge des types de handicap divers.

Dans les institutions où se pratique l'indifférenciation, les trois types de professionnels assument le rôle de référent, qui signifie "être garant de la situation» du résident. Cette responsabilité comprend des activités d'élaboration, de suivi et d'évaluation du «projet individuel » du résident, notamment l'analyse des "besoins de soutien ", la définition des " objectifs » et " moyens » à mettre en place en vue du maintien ou du développement des acquis, la gestion de la situation du résident sur le plan "administratif», l'élaboration des "bilans» et "synthèses» d'accompagnement, le partenariat avec le réseau professionnel et la famille. 
14 Les professionnels ont en outre tous en charge l'accompagnement au quotidien des résidents. Celui-ci implique des "soins de base » ("lever » et " coucher » des résidents, leur " donner à manger, à boire ", " aller aux toilettes avec eux », les aider pour la " douche », etc.), ainsi que des activités relationnelles et éducatives (par exemple, «accueillir les résidents au retour du travail», "parler avec eux de la journée»). Il consiste également en l'organisation et l'accompagnement dans les «tâches d'intendance » (faire «le repas », «la vaisselle ", "le ménage " "en guidance avec les résidents») et "les loisirs " (par exemple, " aller à la piscine », en « promenade »).

15 L'exercice de ces responsabilités et activités mobilise des compétences de la part des trois profils professionnels en matière de référence des résidents : être à même de " construire des projets ", avoir des capacités « d'analyse » de la situation, savoir « poser des objectifs", être en mesure de rédiger des "bilans » et "synthèses » d'accompagnement, "connaître le réseau ", avoir de l'« entregent", etc. Il nécessite aussi des compétences d'accompagnement au quotidien, par exemple, avoir une "expérience d'accompagnement ", des " connaissances » du domaine d'intervention, des habiletés dans la "relation à l'usager». Finalement, il requiert des aptitudes de travail en équipe, par exemple, "pouvoir collaborer", savoir " communiquer avec les collègues" et "utiliser les bons moyens de communication", "se transmettre les informations ", " respecter les décisions prises en équipe ", ainsi que des savoir-être transversaux aux activités comme la "créativité », la «polyvalence», l'« ouverture ».

Dans les institutions qui distinguent sur le plan formel les ASE et les éducateurs sociaux ES et HES, la différence concerne principalement les responsabilités attribuées, cette distinction engendrant quelques variations dans les activités. La référence des résidents revient aux éducateurs sociaux ES et HES: l'élaboration, le suivi et l'évaluation $\mathrm{du}$ projet individuel $\mathrm{du}$ résident sont uniquement $\mathrm{du}$ ressort des personnels de formation supérieure et des compétences plus spécifiques en la matière sont attendues de leur part. L'ASE peut quant à lui exercer comme "coréférent ", son rôle étant alors d' «aider les personnes de référence » dans cette responsabilité et les activités y afférentes. Une distinction s'observe dès lors également dans les positions, de soutien versus garant: l'ASE agirait "par délégation ou sous la responsabilité des éducateurs", ceci impliquant notamment qu'il n'est pas supposé travailler seul. Si des distinctions en matière de références se présentent sur le plan formel entre ASE et éducateurs sociaux ES et HES, l'accompagnement au quotidien concerne cependant les trois groupes professionnels, ceci de la même manière. Il n'y a pas, selon les répondants, de distinction dans les tâches d'accompagnement au quotidien attribuées, ni dans les compétences attendues sur ce plan en fonction du niveau de formation.

\section{L'indifférenciation évite la segmentation, mais nie les différences de formation et de salaire}

La logique de l'indifférenciation est généralement perçue positivement par les directions et personnels qui l'expérimentent. Elle présente l'avantage d'éviter une segmentation et/ou une "hiérarchisation" des activités et professions de l'éducation sociale, perçues comme peu compatibles avec les réalités de l'intervention socioéducative et difficiles à « intégrer » dans le quotidien.

"Donc je pense qu'on ne peut pas réellement séparer le soin de l'éducatif. En fait, c'est une

espèce d'accompagnement global. Dans les soins, c'est aussi des moments qui sont souvent 
éducatifs, parce qu'on est souvent face-à-face avec la personne, c'est aussi des moments où on peut avoir des discussions, des choses un peu intimes. Mais je pense qu'on perdrait en qualité de se dire 'On commence à vraiment séparer ça'. " (Éducateur social ES, homme, 32 ans)

18 Ne pas distinguer contribuerait ainsi, du point de vue de plusieurs interviewés, au maintien d'un « accompagnement global » et de « qualité » des résidents.

Cette logique comporte cependant quelques limites, les principales étant qu'elle ne tient pas compte, sur le plan formel, des différences de niveau de formation et de salaire. Alors que la classe salariale des éducateurs sociaux ES et HES est très proche ou identique selon les cantons, celle des ASE est largement inférieure : ceux-ci sont payés environ $20 \%$ de moins que les éducateurs sociaux. Les divers acteurs interviewés qualifient cette différence de peu "cohérente " et peu "adaptée ", dans un contexte où les trois groupes professionnels partagent un même cahier des charges, des responsabilités et activités prescrites identiques. Des directions et personnels exerçant dans des institutions pratiquant l'indifférenciation émettent en outre le souhait d'une clarification et d'une redéfinition des responsabilités et activités attribuées en fonction des différences de formation. Il s'agit de valoriser chaque groupe professionnel, de favoriser leur complémentarité et de légitimer les différences de niveau de qualification et de salaire.

20 L'indifférenciation, associée à un écart considérable sur le plan salarial entre ASE et éducateurs sociaux ES et HES, soulève un enjeu majeur, celui d'une possible déqualification relative des emplois (Chopart, 2003), soit d'un potentiel remplacement des professionnels de formation supérieure (éducateurs sociaux ES et HES) par des diplômés de niveau inférieur (ASE). Ce risque paraît d'autant plus sérieux dans un contexte de «chalandisation $»^{4} \mathrm{du}$ secteur social (Chauvière, 2014), caractérisé, notamment, par une rationalisation de ses coûts (Bertaux \& Hirlet, 2012).

21 Par ailleurs, soulignons que la professionnalisation du domaine du travail social est un objectif qui a largement été mis en avant afin de promouvoir l'introduction de la formation d'ASE (Perriard \& Castelli Dransart, 2016), la perspective étant en effet que les ASE remplacent progressivement les personnes sans diplôme en travail social qui exercent dans ce champ, dont la part demeure encore importante en Suisse. Dans les institutions résidentielles du handicap, un tiers des personnels n'a pas de formation formelle pour l'activité pratiquée ou est en cours de formation (IWSB, 2016). Or la logique de l'indifférenciation laisse entrevoir que les ASE pourraient relayer non seulement des personnels travaillant sans diplôme, mais également des professionnels de niveau de formation supérieure, ce qui questionne l'objectif initialement affiché d'une amélioration globale du niveau de qualification dans le domaine du travail social.

Quoi qu'il en soit, les résultats de la recherche montrent que les professionnels ne se laissent pas, sur le terrain, enfermer dans le travail prescrit (Bertaux, Schleret \& Bernardi, 2000) et qu'ils développent des logiques professionnelles grâce auxquelles ils s'approprient les secteurs d'intervention formellement attribués, voire les redéfinissent. En effet, une re-répartition des responsabilités et activités s'opère à l'intérieur des équipes, en aval du cahier des charges, qui tient compte des champs d'expertise, de l'«expérience», des "intérêts», mais aussi des "connaissances» et «compétences» spécifiques acquises en formation. Par exemple, plusieurs répondants constatent qu'en dépit d'un cahier des charges unique et d'un espace d'intervention partagé sur le plan formel, les éducateurs sociaux ES et HES tendent à être plus souvent 
" au bureau» que les ASE, occupés à des activités administratives, conceptuelles et organisationnelles. Ensuite, les professionnels de niveaux de formation différents qui " ont tous besoin les uns des autres, mais à d'autres niveaux» s'apportent un soutien mutuel. Ainsi, comme le soulignent des participants, des ASE mobilisent l'appui des éducateurs sociaux ES et HES, par exemple pour la rédaction de bilans d'accompagnement ou l'élaboration de projets individuels, alors que des éducateurs sociaux ES et HES sollicitent l'aide des ASE afin d'être aiguillés dans certaines activités, telles celles d'animation.

En outre, face à la logique de l'indifférenciation, les participants - tous types d'acteurs confondus - s'accordent pour noter des différences dans la «posture» des professionnels, autrement dit dans la manière d'investir leur espace d'intervention. Selon les représentations des répondants, l'ASE est davantage dans la "pratique ", l'« action ", le "concret ", alors que l'éducateur social HES se situe plus dans la "théorie » et la "réflexion». L'éducateur social ES serait "entre deux», constituant le "trait d'union» entre la pratique et la théorie. Les éducateurs sociaux ES et HES sont dès lors perçus par les quatre types d'acteurs interviewés comme ayant une "capacité réflexive» plus large et comme étant mieux outillés sur ce plan par le biais de leur formation.

« Entre moi et HES, oui, HES formé diplômé. Oui, ça on voit quand même qu'il y a plus de

réflexion, qu'ils remettent souvent en question, qu'ils analysent plus que ce que moi j'aurais analysé par exemple. » (ASE, femme, 21 ans) l'apanage de l'éducateur social HES. En référence aux participants, elle signifie, pour le professionnel, avoir une approche "globale», mais aussi approfondie, de la situation, "amener des concepts théoriques" " pour pouvoir plus aller au fond». Elle implique également "de prendre du recul», d'avoir "une faculté d'analyse de la situation", "de comprendre le sens» de l'action et "les enjeux» qui se jouent, "de remettre en question l'intervention ou certaines règles». Ces différences dans la posture professionnelle se traduisent particulièrement dans l'exercice de certaines activités, notamment les tâches d'écriture relatives à l'élaboration, au suivi et à l'évaluation du projet individuel.

"C'est peut-être le niveau de réflexivité et puis je dirais peut-être le... ça se voit souvent dans les écrits, dans la qualité des rapports. Je dis souvent ce terme-là : le niveau de granularité qu'on trouve dans certains projets, dans certains objectifs, dans certains questionnements, dans la manière de gérer les rapports aux représentants légaux. " (Direction, homme, 38 ans).

Elles s'expriment également dans les possibilités de se positionner face au réseau professionnel et non professionnel (médecin, curateur $^{5}$, famille, etc.).

\section{La différenciation : une logique pertinente, mais un risque de taylorisation du travail social}

Pour les directions et professionnels qui l'expérimentent, la logique institutionnelle de la différenciation est pertinente, car elle prend en compte la différence de salaire et de niveau de formation.

« Normal qu'une personne de type ES ou HES qui a fait des études une fois et demie, voire deux fois plus longues que moi ait des responsabilités plus grandes que vis-à-vis de nous. Responsabilité est égale aussi à un salaire plus grand. » (ASE, femme, 26 ans) 


\section{outils" pour assumer les responsabilités spécifiques inhérentes à la référence des} résidents et les activités d'élaboration, de suivi et d'évaluation du projet individuel qui y sont liées. Pour quelques interviewés de formation supérieure toutefois, ces différences formelles - pratiques de conception et conduite du projet individuel des résidents pour les éducateurs sociaux ES et HES, relation directe au résident et tâches de la vie quotidienne pour les ASE - ne feraient pas sens, car elles contribueraient à "découper le métier de l'éducation ».

La logique de la différenciation comporte en effet le risque d'une spécialisation des activités et professions de l'éducation sociale. Ce processus, constaté depuis nombre d'années en France, pays qui compte de longue date des professions de l'éducation sociale de niveaux de formation différents, se caractérise par une "taylorisation» de l'intervention sociale (Libois \& Bolzman, 2014) et un retrait des professionnels plus qualifiés vers les fonctions de "back office » (Ion, 2009). Ce mécanisme va à l'encontre d'une conception classique de l'accompagnement social, qui l'envisage comme une prise en charge globale et entrevoit la relation directe à l'usager comme le cœur de l'intervention sociale (Chopart, 2000) et une condition nécessaire à sa réalisation (Bodin, 2011).

« En tout cas, ce que moi je ne laisserai pas faire, sauf si on m'y oblige à coups de lois, c'est de segmenter le travail social, parce que chacun des gestes du quotidien de l'éducateur est un geste précieux et qui va vraiment l'amener à rencontrer et accompagner la personne. Si vous déléguez à quelqu'un l'hygiène par exemple, mais comment voulez-vous parler avec le résident de son projet de vie?» (Direction, femme, 41 ans)

Dès lors, plusieurs participants pour qui la différenciation est légitime, notent l'importance de maintenir une certaine transversalité entre les responsabilités et activités des professionnels de niveaux de formation différents. Autrement dit, il leur paraît nécessaire, d'une part, que les ASE puissent participer aux activités de référence des résidents et d'autre part, que les éducateurs sociaux ES et HES poursuivent la réalisation d'activités d'accompagnement au quotidien.

"Qu'on n'arrive pas à quelque chose où les ASE, ils font les douches, ils servent les repas, (...), ces choses-là et puis que nous, on n'est plus que dans les projets et puis l'écriture et la construction des projets, et des activités liées aux projets. Je trouve que c'est important que ces choses-là puissent rester partagées. (...). Ben voilà, parce qu'il se passe aussi plein de choses quand on accompagne quelqu'un pour la douche, (...) enfin, je pense qu'on louperait des moments qui peuvent être importants justement dans la construction de projets avec ces gens-là en étant dégagés de ces choses-là. » (Éducateur social ES, homme, 32 ans)

Autre défi lié à ce modèle : son opérationnalisation. Plusieurs directions interviewées notent en effet la difficulté à appliquer les différences formelles aux réalités de la pratique.

«Après, c'est difficile sur le terrain de mettre ça en place, vraiment. » (Direction, femme, 32 ans)

Certains employeurs précisent que, dans leur institution, des ASE sont de fait référents de résidents et travaillent seuls sur le terrain, contrairement à ce que prévoit leur cahier des charges. Diverses raisons sont avancées pour expliquer cet écart entre travail prescrit et réel : un manque d'éducateurs sociaux ES et HES pour assumer la responsabilité de l'ensemble des références; la petite taille des équipes qui empêche de diviser le travail et d'exercer constamment en binôme ASE-éducateur ; les compétences 
des ASE, qui leur permettent, en particulier lorsqu'ils gagnent en expérience, de faire plus que ce que prévoit leur cahier des charges.

\section{Une bonne collaboration, mais des questionnements pour le futur}

Alors que l'augmentation des positions dans l'espace professionnel du travail social (Aballéa, 2000) et son partage entre les différents groupes qui l'occupent semblent généralement source de tensions (Dubar, 2003), les résultats de notre recherche montrent que la cohabitation entre ASE, éducateurs sociaux ES et éducateurs sociaux HES «se passe bien », quelle que soit la logique institutionnelle à l'œuvre en matière de division du travail, indifférenciation ou différenciation. En effet, les qualificatifs les plus fréquemment utilisés par les divers types d'acteurs pour décrire leur expérience de travail dans une équipe composée de personnels issus des trois groupes professionnels ont été: "richesse», "partenariat", "respect», "pluralité", "complémentarité" et "communication». Seuls trois termes avec une connotation négative ont été cités de façon isolée, à savoir " préoccupation ", " prise de tête » et "risque de tension".

La cohabitation entre ASE, éducateurs sociaux ES et éducateurs sociaux HES est perçue par les directions et professionnels comme un plus, aussi bien pour le travail d'équipe que pour l'accompagnement des résidents. La «diversité » et la "complémentarité » des regards, des connaissances spécifiques du métier, des postures, des compétences, des techniques, méthodes et outils professionnels permettraient un apprentissage et un soutien mutuels, ainsi qu'une prise en charge différenciée des résidents. Toutefois, selon les participants, la pluralité des cultures professionnelles pourrait constituer également une limite en l'absence d'un travail d'explicitation et de coordination des informations, cahiers des charges, responsabilités et activités entre les trois profils professionnels.

Deux éléments semblent contribuer au bon fonctionnement actuel de la collaboration : d'une part, une certaine transversalité dans le travail et horizontalité des rapports entre les trois groupes professionnels, d'autre part, un équilibre numérique entre ASE, éducateurs sociaux ES et HES au sein des équipes éducatives.

La transversalité dans le travail et l'horizontalité des rapports apparait, pour les divers acteurs interviewés, comme un plus pour la collaboration.

«Pour le bon fonctionnement d'une équipe et d'un groupe, ça me semble assez important qu'on garde un équilibre là-dedans et puis que ça ne devienne pas quelque chose de très hiérarchisé. » (Éducateur social ES, homme, 32 ans)

Dès lors, une « division morale du travail » (Hughes, 1996), sous la forme d'une délégation, de la part des éducateurs sociaux ES et HES, du «sale boulot» (ibid.), c'est-à-dire des activités considérées comme "ingrates» ou peu valorisées (par exemple, accompagnement aux toilettes, nettoyages) aux ASE, ainsi que des rapports de type vertical sont envisagés par les participants comme dommageables pour la cohabitation et à proscrire.

"Alors il ne faudrait pas qu'il y ait genre 'Parce que moi je suis HES, je fais ça et puis moi je suis ASE et du coup je ne peux pas porter une seule responsabilité'. (Éducateur social ES, femme, 35 ans) 
equipes éducatives, au moment de l'enquête la répartition montre une plus forte représentativité des éducateurs sociaux ES et HES par rapport aux ASE, dont la part est généralement plutôt faible et peut représenter, dans les institutions consultées, au maximum un quart des effectifs. Parmi les éducateurs sociaux, la part des diplômés HES est généralement supérieure à celle des ES (60-70\% versus 40-30\%). Cependant, des directions et des professionnels craignent l'engagement d'ASE qui viendraient relayer non pas les personnels sans diplôme, mais les professionnels ES et HES. Autrement dit, ils font part de leurs inquiétudes quant au remplacement de qualifications établies par des qualifications inférieures, phénomène qui s'est d'ailleurs largement produit en France, en particulier dans le secteur du handicap (Bertaux \& al., 2000 ; Chopart, 2000).

"Je crains aussi qu'on se retrouve avec plus de personnes formées dans le domaine socio-

éducatif ASE et moins de personnes formées HES, surtout HES. " (Direction, femme, 40 ans)

Selon les participants, trois éléments questionnent la possibilité du maintien d'un nombre important de professionnels de formation supérieure au sein des institutions du handicap. Premièrement, les difficultés de recrutement d'éducateurs sociaux ES et HES dans le secteur du handicap, évoquées par plusieurs directions. Ces difficultés relèveraient d'un manque d'attractivité du domaine lié, d'une part, à la pénibilité d'un travail qui nécessite beaucoup de soins de base, notamment dans les champs du handicap physique et du polyhandicap ; d'autre part, aux problématiques rencontrées, par exemple le vieillissement des résidents et la régression de leurs capacités. Deuxièmement, l'incertitude concernant les ressources et contraintes financières futures des institutions liées à leur subventionnement.

"J'espère qu'au niveau économique, avec les restrictions budgétaires qui se font ces tempslà, que les HES, on ne va pas être mis de côté au profit d'engagements d'ASE, parce que c'est vrai qu'ils coûtent moins cher. » (Éducateur social HES, femme, 29 ans)

41 Finalement, les directions et personnels s'interrogent sur les directives cantonales à venir en matière de répartition des profils professionnels et font part de leurs inquiétudes quant à la potentielle introduction de politiques de quotas, si ces dernières devaient limiter le nombre de professionnels de formation supérieure. Pour l'instant, il n'y a pas, dans les cantons concernés, de directives strictes règlementant la proportion de professionnels ASE, éducateurs sociaux ES et HES dans les structures résidentielles $\mathrm{du}$ handicap. Les autorités cantonales de surveillance des établissements du handicap émettent seulement des recommandations, qui encouragent les institutions à privilégier une logique de répartition fondée sur les besoins des résidents et leur prise en charge. 


\section{Conclusion} formation différents, leur définition théorique en trois profils professionnels distincts et la division du travail telle qu'elle s'opère dans les établissements suisses romands du handicap. Le constat de ce décalage peut à première vue questionner quant à une possible compatibilité entre le modèle actuel de formation et les modèles institutionnels d'organisation du travail. Cependant, en Suisse, la reconfiguration de la formation et des professions de l'éducation sociale est récente et la situation susceptible d'évoluer. En effet, notre étude a été menée alors que les nouveaux diplômés venaient d'arriver sur le marché du travail et que l'expérience de l'articulation entre ces groupes professionnels sur les terrains était très courte. Un certain recul par rapport aux récentes transformations contribuera sans doute à des ajustements de part et d'autre qui permettront de réduire la distance. A cette fin, la poursuite de discussions et réflexions, menées entre les acteurs des milieux professionnels, des employeurs, de la formation et de la recherche, est à encourager. Afin de soutenir ces réflexions, il serait par ailleurs utile d'élargir la recherche sur la 
collaboration à d'autres groupes professionnels (par exemple, les assistants en soins et accompagnement), domaines du travail social (par exemple, enfance, personnes âgées) ou encore à d'autres pays ayant un système de formation en travail social comportant plusieurs niveaux de qualification, afin d'explorer d'éventuels autres modes de fonctionnement et d'organisation.

Contrairement à certains constats, selon lesquels la multiplication des groupes professionnels est problématique pour leur collaboration (Aballéa, 2000 ; Dubar, 2003), la cohabitation entre ASE, éducateurs sociaux ES et HES dans les structures résidentielles du handicap fonctionne bien, grâce à une transversalité dans le travail, une horizontalité des rapports et un certain équilibre, au sein du personnel formé, entre ces groupes.

Cependant, le recrutement de personnel formé, en particulier au niveau supérieur, pose problème dans le secteur du handicap, comme l'a montré notre recherche. Or, ce secteur est actuellement traversé par des défis majeurs, tels le vieillissement de sa population ou la complexification des situations de handicap, qui nécessitent une prise en charge de qualité, assurée par un personnel qualifié. L'engagement d'un nombre accru d'ASE afin de relayer les personnels non formés en travail social, encore nombreux dans le secteur du handicap, est à soutenir, de même que la formation de ces personnels non diplômés à la profession d'ASE ou à d'autres métiers (par exemple celui d'ASA). Cependant, une présence importante d'éducateurs sociaux ES et HES doit également pouvoir être garantie afin de maintenir des prestations de qualité, ces professionnels étant mieux préparés pour assurer des tâches exigeantes et complexes. À ce titre, une valorisation du secteur du handicap au sein des formations supérieures, passant notamment par une meilleure connaissance de ce champ et des problématique spécifiques actuelles qui l'animent, paraît souhaitable.

La recherche sur laquelle s'appuie cet article offre une première photographie de la collaboration entre ASE, éducateurs sociaux ES, éducateurs sociaux HES dans les institutions du handicap en Suisse romande, et de ses enjeux. Ses résultats doivent cependant être généralisés et interprétés avec précaution, d'abord en raison de la taille relativement limitée de l'échantillon, de sa non-représentativité et de sa constitution sur la base du volontariat. Ensuite, les éléments relevés concernant les logiques institutionnelles, le positionnement des acteurs face à ces logiques et la collaboration reposent sur le discours des participants. Des observations in situ et l'analyse de documents institutionnels (cahiers des charges, descriptifs de fonctions, etc.) auraient sans doute contribué à compléter et nuancer certains résultats. Finalement, la recherche a analysé la collaboration entre les trois groupes professionnels à un moment donné, mais n'a pas permis d'en suivre l'évolution afin de mettre en perspective les différentes tendances.

Les groupes professionnels étant "évolutifs » (Demazière \& Gadéa, op. cit., p. 20) et leur transformation liée à celle des contextes dans lesquels ils se construisent (Conq, 2010), il serait intéressant de reconduire, d'ici quelques années, une recherche sur ce thème. $\mathrm{Au}$ vu du contexte d'instabilité dans lequel la recherche a été menée, mais aussi des incertitudes, notamment d'ordre économique et politique, qui planent sur les institutions du handicap, et plus globalement sur le champ du travail social, l'arrêt sur image fourni par cette nouvelle étude pourrait s'avérer sensiblement différent du présent cliché. 


\section{BIBLIOGRAPHIE}

Aballéa F. (2000), « Quel avenir pour les professions installées ? », in Chopart J.-N. (sous la dir. de), Les mutations du travail social. Dynamiques d'un champ professionnel, Paris, Dunod, pp. 97-110.

Abbot A. (1988), The System of the Professions. An Essay of the Division of Expert Labour, Chicago, Chicago University Press.

Bardin L. (2007), L'analyse de contenu, Paris, PUF.

Bercot R. ; Divay S. ; Gadea C. (2012), Les groupes professionnels en tension, Toulouse, Octares.

Bertaux R. ; Hirlet P. (2012), « Les acteurs de l'intervention sociale entre hétéronomie et autonomie du travail », Vie sociale, $\mathrm{n}^{\circ}$ 1, pp. 157-172.

Bertaux R. ; Schleret Y. ; Bernardi S. (2000), « Logiques professionnelles, logiques institutionnelles, logiques de mission », in Chopart J.-N. (sous la dir. de), Les mutations du travail social. Dynamiques d'un champ professionnel, Paris, Dunod, pp. 215-228.

Bodin R. (2011), « Une éducation sentimentale. Sur les ambiguïés de l'accompagnement social en éducation spécialisée », Déviance et Société, $\mathrm{n}^{\circ}$ 35, pp. 93-112.

Chauvière M. (2014), « Nouvelles représentations du travail social. Ses enjeux en France », in Voélin S. ; Eser Davolio M. ; Lindenau M. (sous la dir. de), Le travail social entre résistance et innovation, Genève, Editions IES.

Chopart J.-N. (2000), « Conclusion : du travail social à l'intervention sociale », in Chopart J.-N. (sous la dir. de), Les mutations du travail social. Dynamiques d'un champ professionnel, Paris, Dunod, pp. 267-274.

Chopart J.-N. (2003), «Retour réflexif sur un programme de recherche : que fait la sociologie des professions face à la marchandisation du champ social ? ", in Vilbrod A. (sous la dir. de), L'identité incertaine des travailleurs sociaux, Paris, L'Harmattan, p. 39-55.

CDIP Conférence suisse des directeurs cantonaux de l'instruction publique (1999), Décisions concernant les formations dans le domaine du travail social, 26 août 1999. Récupéré de http:// edudoc.ch/record/25518/files/19990826B_f.pdf.

Conq N. (2010), « Introduction », in Conq N., Kervella J.-P., Vilbrod A. (sous la dir. de), Le métier d'éducateur spécialisé à la croisée des chemins, Paris, L'Harmattan, pp. 11-19.

Demazière D. ; Gadéa C. (sous la dir. de) (2009), Sociologie des groupes professionnels. Acquis récents et nouveaux défis, Paris, La Découverte.

Dubar C. (2003), « Les travailleurs sociaux, un groupe professionnel ? », in Vilbrod A. (sous la dir. de), L'identité incertaine des travailleurs sociaux, Paris, L'Harmattan, pp. 17-25.

Dubar C. ; Tripier P. ; Boussard V. (2011), Sociologie des professions (3 $3^{\mathrm{ème}}$ éd.), Paris, Armand Colin.

Hughes E. C. (1996), Le Regard sociologique, Paris, Editions de l'EHESS.

IWSB Institut d'études économiques de Bâle (2016), Demande de personnel qualifié et besoin de formation dans le champ du travail social. Un aperçu des différentes professions sociales et domaines d'activités, Olten, Savoir social.

Ion J. (2009), « Travailleurs sociaux, intervenants sociaux : quelle identité de métier ? ", Informations sociales, $\mathrm{n}^{\circ}$ 152, pp. 136-142. 
Keller V. (2017), La campagne nationale d'AvenirSocial : une formation en travail social pour des prestations de qualité. Document de référence, Berne, AvenirSocial.

Libois J. ; Bolzman C. (2014), « Processus de disciplinarisation du travail social : le cas de la Suisse ", in Jaegger M. (sous la dir. de), Le travail social et la recherche, Paris, Dunod.

Miles M.B. ; Huberman A.M. (2003), Analyse des données qualitatives ( $2^{2 \mathrm{eme}}$ éd.), Bruxelles, De Boeck.

Perriard V. ; Castelli Dransart D.A. (2016), « La nouvelle profession d'assistant socio-éducatif (ASE) en Suisse romande : quels développements et enjeux pour le champ professionnel du travail social ?", Travail-Emploi-Formation, n 14, pp. 76-89.

Schön D.A. (1983), The reflective Practitioner, New York, Basic Book.

\section{NOTES}

1. La notion d'éducation sociale renvoie à celle d'éducation spécialisée utilisée en France.

2. Précisons que ces professionnels sont susceptibles de collaborer, au sein des structures du handicap, avec des diplômés en travail social ou en éducation sociale titulaires d'autres titres et formations, par exemple des aides en soins et accompagnement (attestation fédérale professionnelle d'ASA). Ils exercent également avec des personnels non diplômés dans le domaine, dont le nombre, en Suisse, reste important, en particulier dans le secteur du handicap (IWSB, 2016). Afin de limiter le champ de la recherche, notre étude s'est toutefois focalisée sur la collaboration entre ASE, éducateurs sociaux ES et éducateurs sociaux HES, les trois professions étant présentes dans les structures résidentielles du handicap de tous les cantons romands. En outre, les diplômés ASA n'étaient pas encore en exercice au moment de l'enquête.

3. Les passages entre guillemets renvoient à des propos extraits des entretiens avec les participants de la recherche.

4. L'entrée de la logique du marché dans le champ social.

5. Dans le Code civil suisse (Art. 390), « Le curateur assiste et représente les personnes sous curatelle. La curatelle est une mesure de protection, volontaire ou imposée par l'État (autorité de protection de l'adulte) qui est instituée lorsqu'une personne: est partiellement ou totalement empêchée d'assurer elle-même la sauvegarde de ses intérêts en raison d'une déficience mentale, de troubles psychiques ou d'un autre état de faiblesse qui affecte sa condition personnelle; est, en raison d'une incapacité passagère de discernement ou pour cause d'absence, empêchée d'agir elle-même et n'a pas désigné de représentant pour des affaires qui doivent être réglées. » Source: https://www.droit-bilingue.ch/rs/lex/1907/00/19070042-a390-frde.html

\section{RÉSUMÉS}

Suite aux réformes de la formation en travail social, cohabitent dans les institutions du handicap en Suisse romande des professionnels de l'éducation sociale de niveaux de qualification différents : assistants socio-éducatifs (ASE), éducateurs sociaux diplômés d'une école supérieure (ES) et éducateurs sociaux titulaires d'un Bachelor d'une haute école spécialisée (HES). Les entretiens mettent en évidence deux logiques institutionnelles de division du travail qui se 
développent dans les structures consultées, indifférenciation et différenciation, les enjeux qu'elles soulèvent, ainsi que la manière dont les professionnels se les approprient.

Following reforms of the social work education system, social education professionals with different levels of diplomas now work together in institutions for persons with disabilities in French-speaking Switzerland: socio-educational assistants (SEA), social educators with an Advanced Federal Diploma of Higher Education (SEH) and social educators with a Bachelor of Arts (SEU). Interviews point out two institutional modalities of division of labour in the consulted structures, indifferentiation and differentiation, the issues they raise and how professionals make them their own.

Zusammenfassung : Die Ausbildungsreform im Bereich der sozialen Arbeit haben bewirkt, dass in den Westschweizer Behinderteneinrichtungen Fachkräfte mit unterschiedlichen Abschlüssen arbeiten: Fachpersonen Betreuung (FaBe), Sozialpädagogen Höhere Fachschule (HF) und Sozialpädagogen Fachhochschule (FH). Anhand von Interviews wird aufgezeigt, dass sich die Arbeitsteilung in den betrachteten Einrichtungen nach zwei Gesichtspunkten gestaltet, nämlich Undifferenziertheit und Differenziertheit, welche Fragen und Spannungen damit verbunden sind und wie sie von den Fachkräften aufgegriffen wird.

Como resultado de las reformas de la formación en trabajo social, en las instituciones para discapacitados de la Suiza francófona cohabitan profesionales de educación social de diferentes niveles de calificación: asistentes socioeducativos (ASE), educadores sociales con educación superior (ES) y educadores sociales con un grado de Bachelor de una alta escuela especializada (HES). Las entrevistas destacan dos lógicas institucionales de división del trabajo que se desarrollan en las estructuras consultadas, indiferenciación y diferenciación, los problemas que plantean, así como la forma en que los profesionales se las apropian.

\section{INDEX}

Palabras claves : asistente social, educador especializado, enseñanza técnico-profesional, organización del trabajo, división del trabajo, nivel de formación, minusvalidez, Suiza

Mots-clés : assistant social, éducateur spécialisé, enseignement technique-professionnel, organisation du travail, division du travail, niveau de formation, handicap, Suisse

Schlüsselwörter : Sozialhelfer, Sonderschulerzieher, Gewerblich-Technisches Ausbildungswesen, Arbeitsorganisation, Arbeitsteilung, Ausbildungsstufe, Behinderung, Schweiz Keywords : social worker, special education teacher, work organisation, division of labour, training level, disability, Switzerland

Code JEL I12 - Health Production: Nutrition; Mortality; Morbidity; Substance Abuse and Addiction; Disability; and Economic Behavior, M54 - Labor Management (team formation; worker empowerment; job design; tasks and authority; job satisfaction)

\section{AUTEURS}

\section{VALÉRIE PERRIARD}

Professeure à la Haute école de travail social Fribourg (HETS-FR), Haute école spécialisée de Suisse occidentale (HES-SO) 


\section{ALIDA GULFI}

Professeure à la Haute école de travail social Fribourg (HETS-FR), Haute école spécialisée de Suisse occidentale (HES-SO) 